\title{
Consumo alimentar de adolescentes: Validação e calibração de um questionário de frequência alimentar em estudo com amostragem complexa
}

\author{
Adolescent food intake: Validation and calibration of a food frequency questionnaire in a complex \\ sampling study \\ Consumo de alimentos en adolescentes: Validación y calibración de un cuestionario de frecuencia \\ alimentaria en un estudio con muestreo complejo
}

\section{Resumo}

Estudos sobre padrões alimentares dependem da validade dos métodos, como o questionário de frequência alimentar (QFA), para estimar consumo alimentar de longo prazo, que pode ser superestimado e o método precisa ser validado. Questiona-se se estudos de validação aninhados a estudos com desenho amostral complexo (DAC) deveriam ignorar a dependência dos dados. Este estudo objetiva validar o QFA aplicado a adolescentes escolares e estimar os fatores de calibração em um estudo com amostragem complexa. A validade do QFA é testada frente a registros alimentares (RA) aplicados a 85 adolescentes em um estudo com DAC, com estimação dos fatores de calibração $(\lambda)$ para energia, nutrientes e grupos alimentares (GA). Coeficiente de correlação intraclasse foi calculado; diferenças médias foram comparadas via modelagem, e foram realizadas análises de concordância. As médias entre os métodos diferiram para energia e nutrientes $(\mathrm{p}<0,05)$, mas quatro GA foram similares. Limites de concordância não se mostraram aceitáveis para os nutrientes. Coeficientes de correlação de concordância de fibras, zinco e ácidos graxos, ajustados pela energia, variaram de 0,16 a 0,22 e para os GA variaram de 0,07 a 0,36. Os fatores de atenuação corroboraram as diferenças $(\lambda<0,40)$. O QFA apresentou validade relativa, sobretudo para os GA e $\lambda$ aproximou as estimativas do QFA ao RA, sendo relevante quando nutrientes são usados em análises epidemiológicas. O ajuste das análises pelo DAC confirmou que a estrutura de dependência dos dados deve ser considerada, mesmo em estudos de validação, devendo-se evitar tratar tais dados como se fossem oriundos de amostra aleatória simples.

Palavras-chave: Adolescentes; Questionário de frequência alimentar (QFA); Validação; Calibração; Amostragem complexa.

\begin{abstract}
Studies on dietary patterns depend on the validity of methods, such as the food frequency questionnaire (FFQ), to estimate long-term food intake, which can be overestimated, and the method needs to be validated. It is questioned whether validation studies nested in studies with complex sample design (CSD) should ignore data dependence. This study aims to validate the FFQ applied to school adolescents and estimate the calibration factors in a study with complex sampling. The validity of the FFQ is tested against dietary records (DR) applied to 85 adolescents in a study with CSD, with estimation of calibration factors $(\lambda)$ for energy, nutrients, and dietary groups (DG). Intraclass correlation coefficient was calculated; mean differences were compared by modeling, and agreement analyzes were performed. The means between the methods differed for energy and nutrients $(p<0.05)$, but four DG were similar. Limits of agreement were not acceptable for nutrients. Correlation coefficients of fiber, zinc, and fatty acids, adjusted for energy, ranged from $0 ., 16$ to $0, .22$ and for DG ranged from 0.07 to 0.36 . The attenuation factors corroborated the differences $(\lambda<0,40)$. The FFQ presented relative validity, especially for DG, and $\lambda$, which brought the FFQ estimates to FR, being relevant when nutrients are used in epidemiological analyzes. The adjustment of the analyzes by the CSD
\end{abstract}


confirmed that the structure of data dependence must be considered, even in validation studies, and it should be avoided to treat such data as if they came from a simple random sample.

Keywords: Adolescents; Food frequency questionnaire (FFQ); Validation; Calibration; Complex sampling.

\section{Resumen}

Los estudios sobre patrones alimentarios dependen de la validez de métodos, como el cuestionario de frecuencia alimentaria (CFA), para estimar el consumo de alimentos a largo plazo, que puede estar sobreestimado y el método debe ser validado. Se cuestiona si los estudios de validación anidados en estudios con diseño de muestra complejo (DMC) deberían ignorar la dependencia de los datos. Este estudio tiene como objetivo validar el CFA aplicado a estudiantes adolescentes y estimar los factores de calibración en un estudio con muestreo complejo. La validez del CFA se contrasta con los registros de alimentos (RA) aplicados a 85 adolescentes en un estudio con DMC, con estimación de los factores de calibración $(\lambda)$ para energía, nutrientes y grupos de alimentos (GA). Se calculó el coeficiente de correlación intraclase; las diferencias medias se compararon mediante modelos y se realizaron análisis de concordancia. Las medias entre los métodos difirieron para la energía y los nutrientes $(\mathrm{p}<0.05)$, pero cuatro GA fueron similares. Los límites de concordancia no se mostró aceptables para los nutrientes. Los coeficientes de correlación de concordancia de fibras, zinc y ácidos grasos, ajustados por energía, variaron de 0,16 a 0,22 y para GA variaron de 0,07 a 0,36 . Los factores de atenuación corroboraron las diferencias $(\lambda<0,40)$. El CFA presentó validez relativa, especialmente para GA y $\lambda$ aproximó las estimaciones de CFA al RA, siendo relevante cuando se utilizan nutrientes en análisis epidemiológicos. El ajuste de los análisis por parte del DMC confirmó que la estructura de dependencia de los datos debe ser considerada, incluso en estudios de validación, evitando tratar dichos datos como si procedieran de una muestra aleatoria simple.

Palabras clave: Adolescentes; Cuestionario de frecuencia alimentaria (CFA); Validación; Calibración; Muestreo complejo.

\section{Introdução}

Os padrões alimentares são importantes fatores determinantes para a ocorrência de diversos agravos à saúde, especialmente as doenças crônicas (Bechthold et al., 2019) e existem evidências na literatura que hábitos e comportamentos adotados na infância e adolescência perduram na vida adulta (WHO, 2005). Entretanto, sua investigação em populações não se constitui em tarefa simples, pois sendo construto não diretamente observado, os padrões alimentares são estimados por métodos de mensuração do consumo alimentar de longo prazo, em substituição ao consumo habitual, que necessitam de validação para sua adequada utilização (Cade et al., 2004; Thompson \& Subar, 2013). Em grandes estudos populacionais, dado a dificuldade de utilização dos métodos referência que mais se aproximam do consumo alimentar usual, como biomarcadores, recordatório 24 horas e registros alimentares (RA), frequentemente utiliza-se o questionário de frequência alimentar (QFA) como representante desse consumo habitual de longo prazo, por ser de fácil e econômica aplicação (Cade et al., 2004; Kipnis et al., 1999) e recentemente em versões digitais auto-administradas (Kouvari et al., 2020).

Porém, as estimativas obtidas no QFA se mostram superestimadas, quando comparadas aos métodos referências (Gigante et al., 2010; Matos et al., 2012), devido a erros de medida inerentes ao próprio QFA, às dificuldades relacionadas à memória do consumo alimentar, ou a efeitos externos, resultando em erro frequentemente maior que outras exposições de interesse epidemiológico, demandando a avaliação da estrutura dos erros de dados de consumo alimentar com abordagens analíticas adequadas (Beaton, Burema \& Ritenbaugh, 1997; Freedman et al., 2011). Uma alternativa para minimizar as limitações do QFA é a realização de estudos de validação e calibração, que testam o grau com que se mensura o consumo alimentar, de acordo com a população do estudo e o erro envolvido (Cade et al., 2004; Beaton, Burema \& Ritenbaugh, 1997; Rutishauser, 2005), e corrigem os erros de medida, aproximando as estimativas do QFA às dos métodos referência utilizados (Slater et al., 2007), a exemplo dos RA que não dependem da memória do respondente (Baranowski, 2012), podendo ser aplicados a adolescentes escolares do ensino médio (Mascarenhas et al., 2016).

No Brasil, poucos estudos de validação foram realizados com adolescentes, quando comparados com a população adulta (Matos et al., 2012; Mascarenhas et al., 2016; Henn et al., 2010; Voci et al., 2011; Brito et al., 2017; Araujo, Yokoo \& Pereira, 2010) e menos ainda no nordeste brasileiro. No estado da Bahia somente dois estudos foram encontrados (Matos et 
al., 2012; Mascarenhas et al., 2016), ambos na capital do estado. Além disso, vários estudos consideram o desenho amostral complexo, utilizando a escola ou área geográfica como conglomerado (Matos et al., 2012; Mascarenhas et al., 2016; Henn et al., 2010; Voci et al., 2011), o que exige métodos de análise adequados. Apesar disso, os estudos de validação e calibração de QFA encontrados na literatura não utilizaram metodologias corrigindo a dependência dos dados, sendo recente a incorporação de ferramentas de análise para amostragem por conglomerados nos softwares estatísticos, que permitem obter estimativas válidas e corrigidas pelo efeito de desenho (Bell et al., 2012). Ignorar o efeito do cluster leva a erros na precisão das estimativas e na significância estatística dos correspondentes testes de hipóteses, resultando na subestimação dos erros padrão e inferência incorreta (Hedges, 2007; Lin et al., 2012) e a análise de dados empíricos permite esclarecer e delimitar a questão (Köche, 2011) no âmbito dos estudos de validação. Assim, os objetivos deste estudo são: (1) avaliar a validade de um QFA aplicado a adolescentes escolares de municípios do interior baiano, e (2) estimar os fatores de calibração usados para a correção das medidas de consumo de nutrientes e grupos alimentares, considerando o efeito do desenho do estudo com amostragem em conglomerados.

\section{Estratégia Metodológica}

Trata-se de um estudo quantitativo, de validação, ancorado em um ensaio comunitário randomizado controlado, integrante do projeto "Desenvolvimento e avaliação de uma intervenção intersetorial no âmbito da Estratégia da Saúde da Família (ESF) sobre padrões alimentares e de atividade física de adolescentes", nominado Prose (Promovendo Saúde na Escola). Para este estudo utilizou-se dados da linha de base do Prose que implementou uma intervenção em escolas públicas do ensino médio de dez municípios que atenderam aos critérios de seleção: entre 20.000 e 60.000 habitantes; cobertura da ESF superior a 90\%; Programa Saúde na Escola implantado em pelo menos duas escolas; e presença de educador físico e nutricionista do Núcleo de Apoio à Saúde da Família. Os estudantes do primeiro e segundo ano do ensino médio, matriculados em vinte escolas (duas por município), constituíram a unidade de análise do Prose.

O cálculo amostral do Prose considerou nível de significância de 0,05 , poder estatístico de 0,80 , efeito de desenho 2,1 , coeficiente de correlação intraclasse para IMC (Índice de Massa Corporal) nas escolas de 0,011 (Richmond \& Subramanian, 2008) e um tamanho de conglomerado médio de 100 estudantes por cluster (escola). Com aumento de 10\% para compensar eventuais perdas no seguimento, o tamanho final da amostra foi estimado, utilizando a fórmula para ensaios randomizados controlados com razão 1 para intervenção/controle (Bland, 2004), em 2.466 sujeitos (1233 no grupo controle e 1233 no grupo de intervenção). Cálculos realizados no software GPower v.3.1.3.

\section{População do estudo de validação e calibração}

Para a seleção da amostra deste estudo, todos os adolescentes da primeira escola visitada no município foram convidados a preencher, de forma voluntária, três dias de RA (dois dias da semana alternados e um do final de semana) e orientados sobre o preenchimento. Dentre os que aceitaram participar (113), foram excluídos 28 adolescentes, quatro por não preencherem os três RA, um por não ter completado o QFA e 23 que relataram consumo energético implausível $(<500$ kcal ou $>7000 \mathrm{kcal}$ ). A subamostra de 85 adolescentes atende as recomendações da literatura para estudos de validação (no mínimo 50 a 100 participantes e oriundos da população do estudo) (Cade et al., 2004; Voci et al., 2011) e obteve-se maior proporção de resposta aos RA nos dias de semana $(66,3 \%)$.

\section{Procedimentos $e$ instrumentos de coleta de dados}

Entre março a abril de 2013 foi aplicado o QFA e coletados os RA. Para minimizar erros de medida sistemáticos e aleatórios, mecanismos de controle foram realizados. Para evitar que a coleta de dados por um método influenciasse as 
respostas do outro (Cade et al., 2004), os RA foram entregues aos adolescentes após aplicação do QFA.

Um QFA com 145 itens alimentares, adaptado inicialmente a partir de dois estudos (Furlan-Viebig \& Pastor-Valero, 2004; Araujo et al., 2010), foi ajustado considerando os hábitos de consumo regionais (frutas, cárneos, e preparações diversas), ficando estruturado com cinco questões: $1^{\mathrm{a}}$ ) Nos últimos 06 meses consumiu "alimento"? 2 $2^{\mathrm{a}}$ ) Qual a frequência com que consome (raramente, mensal, semanal, diário)? $3^{\mathrm{a}}$ ) Quando come esse alimento, quantas vezes você consome ao dia (1 a 5 ou mais vezes/dia)? 4 $4^{\mathrm{a}}$ ) Considerando as medidas caseiras apresentadas, qual a quantidade que você come (1 a 5 ou mais vezes/dia)? e $5^{\text {a }}$ ) Qual o tamanho desta porção (medida caseira), quando aplicável?. A aplicação foi realizada com auxílio de tablets e os dados sincronizados diariamente utilizando o programa RedCAP. Os adolescentes foram orientados para o preenchimento dos RA (porções, horários, alternância dos dias) e receberam álbum contendo porções, utensílios e volume para facilitar a identificação das medidas caseiras e minimizar o viés de informação.

As variáveis sexo e idade foram autorreferidas no questionário sobre hábitos de vida. Peso e altura foram aferidos em duplicata por equipe capacitada, em ambiente reservado em cada escola e de forma padronizada (Lohman, Roache \& Martorell, 1992). O estado antropométrico foi calculado através do IMC (peso(kg)/estatura(m)2), adotando-se os percentis propostos por Onis e colaboradores (2007).

Consumo alimentar. Atribuiu-se pesos às cinco categorias de frequência de consumo do QFA ( $0=$ nunca/raramente; 0,07=1-3 vezes/mês; $0,21=1-2$ vezes/semana; $0,5=3-4$ vezes/semana; $1=5-7$ vezes/semana), com base no estudo de Fornés e colaboradores (2002) e nas características deste estudo, de forma que a frequência de consumo de cada item alimentar fosse tratada como variável quantitativa. Exemplificando, se o adolescente referiu o consumo de 1 a 3 vezes por mês de determinado alimento, o intervalo de frequência variou entre 6 (a) e 18 (b) dias, considerando os seis meses precedentes à aplicação do QFA (181 dias no período do estudo). Dados aplicados na equação para definir os pesos: [Peso=(1/181)*(a+b)/2]. Em seguida, obteve-se o consumo diário para cada item alimentar $(\mathrm{g} / \mathrm{mL})$ multiplicando-se os pesos pelo número de vezes de consumo, quantidade e o tamanho (ou unidade) das porções.

As porções (QFA e RA) foram baseadas em tabelas de medidas caseiras (Pinheiro et al., 2005; IBGE, 2011) e rótulos de alimentos. Para os itens regionais utilizou-se dados do Núcleo de Nutrição e Epidemiologia (Universidade Federal da Bahia - UFBA) e IBGE (2011). Receitas padronizadas foram elaboradas para preparações referidas nos RA que se diferenciaram das contidas no material de apoio distribuído e nas bases de dados dietéticos (tabelas (Pinheiro et al., 2005; IBGE, 2011; Taco, 2011) ou Programa Virtual Nutri 1.0). Também foi apresentada a conversão para consumo diário $(\mathrm{g} / \mathrm{mL})$ para cada item alimentar dos RA.

Os métodos foram analisados através de dois conjuntos de variáveis: a) consumo de energia e nutrientes (macronutrientes, fibras, cálcio, sódio, zinco, ácidos graxos saturados (AcGS)), calculados utilizando-se tabelas brasileiras de composição de alimentos30,31 e o Programa Virtual Nutri 1.0; e b) consumo de vinte grupos alimentares (GA) (g/mL/dia), constituídos com base em características nutricionais e hábitos de consumo. Os alimentos também foram agrupados em: in natura, processados e ultraprocessados, conforme Louzada e colaboradores (2015) considerando as características e de processamento dos itens alimentares.

\section{Análise dos dados}

As variáveis categóricas foram descritas através de distribuições de frequência, e foram utilizadas médias e desvio padrão (DP) para as contínuas. Avaliou-se o padrão de distribuição das variáveis de consumo alimentar (particularmente simetria/normalidade) através de técnicas gráficas. Utilizou-se testes estatísticos para avaliar distribuição de normalidade univariada (Shapiro Wilk) ou bivariada (Doornik-Hansen), suposições requeridas em algumas das análises. Quando a suposição de normalidade da distribuição não foi verificada, considerou-se uso de transformações. A transformação raiz 
quadrada foi a que melhor se adequou para aproximar a distribuição normal.

Correção pelo efeito de desenho. Para estimar a magnitude do efeito do desenho amostral sobre o consumo alimentar dos adolescentes, segundo os RA, utilizou-se o modelo linear misto não condicional, ou seja, o modelo de interceptos aleatórios, sem inclusão de covariáveis. O objetivo do ajuste desse modelo é obter a estimativa para o coeficiente de correlação intraclasse ( $\rho$ ou ICC=Intraclass Correlation Coefficient, em inglês), que permite verificar a distribuição da variância total da variável resposta pelos níveis de agrupamento: estudante e escola, sendo possível identificar qual parte da variância total pode ser atribuída a cada nível (Hedges \& Hedberg, 2007). Para identificar a variância dos estimadores, os ICC obtidos foram substituídos na fórmula do deff (design effect) $=[1+(\mathrm{m}-1)$ ICC $]$, onde $\mathrm{m}$ é o número médio de indivíduos por cluster. O ICC é uma proporção, de modo que quanto mais distante de zero mais forte é a estrutura de dependência dos dados. Neste caso, $\rho$ representa uma medida do efeito do cluster sobre os dados (Hedges, 2007).

Neste estudo, o ICC foi obtido para o consumo alimentar de energia, nutrientes e GA, obtido nos RA, transformados, brutos, e ajustados pela energia (Willett, Howe \& Kushi, 1997) (nutrientes). Em estudos com amostragem complexa como este, a variância da variável resposta é maior do que a obtida em amostras aleatórias simples pelo fator chamado de efeito de desenho por Kish (1965) apud Hedges \& Hedberg (2007), que é função do ICC. Todas as análises para validação e calibração do QFA foram realizadas considerando a dependência dos dados.

Ajuste pela energia. Os nutrientes foram ajustados pela energia através do método dos resíduos (Willett, Howe \& Kushi, 1997) considerando-se os resíduos da regressão linear para a ingestão dos nutrientes, sendo o consumo energético total (Kcal) a variável independente. Como os resíduos incluem valores negativos, a estes foi adicionado o consumo de energia média da amostra (Willett, Howe \& Kushi, 1997), substituindo-se a variável dependente pelos resíduos da regressão, removendo-se assim a variação externa causada pelo consumo de energia total (Kipnis et al., 2003).

Validação do QFA. O consumo de nutrientes e GA, obtidos nos dois métodos, foi comparado através de cinco procedimentos de análise. No primeiro calculou-se o percentual de concordância através da classificação dos adolescentes de acordo com o consumo dos GA em: consumidores (consumo concomitante nos dois métodos, independente da quantidade) e não consumidores (sem consumo nos dois métodos). Definiu-se ainda o percentual de omissão (consumidores no RA, mas não no QFA) e de intrusão (consumidores no QFA, mas não no RA) (Vereecken et al., 2005a).

A segunda análise avaliou a concordância do consumo de energia e nutrientes por meio da construção dos limites de concordância a 95\% (LDC95\%), conforme o método de Bland \& Altman (1986), obtidos a partir da média e DP das diferenças. As diferenças médias (QFA-RA) representam o viés $(d)$, ou seja, as diferenças sistemáticas entre os métodos. Se estas são normalmente distribuídas, espera-se que $95 \%$ das diferenças fiquem entre $d+1,96 \mathrm{DP}$, chamados LDC95\%. Somente para esta análise, os dados foram transformados usando logaritmo, seguindo recomendação da literatura (Bland \& Altman, 1986). Para se obter a interpretação na escala original foi usada a função inversa do logaritmo (anti log). Devido ao uso de transformação logarítmica, a diferença média estimada para cada item avaliado, após o uso da função anti log, expressa a razão geométrica entre as médias de consumo nos dois questionários, que é denominada por concordância média entre os métodos. Os resultados são geralmente multiplicados por 100 e expressos em percentual, de modo que 1 (na escala original) ou 100\% indicam concordância exata (Ambrosini et al., 2003; Brito et al., 2017). Os LDC95\% foram considerados aceitáveis quando ficaram entre $50 \%$ e $200 \%$ (Ambrosini et al., 2003).

A terceira análise utilizou a estatística Kappa para comparar o consumo, usando o método bootstrap para construção dos intervalos de confiança e correção pelo efeito do conglomerado. Os pontos de corte para definir as categorias analisadas foram baseados nos tercis das distribuições dos nutrientes (RA e QFA), ajustados pela energia (Landis \& Koch, 1977; Kang et al., 2013).

A quarta análise testou a hipótese de diferenças das médias entre os métodos via o ajuste de modelo linear misto sem 
covariáveis, tendo como desfecho as diferenças entre os dois métodos (QFA-RA). Assim, a não rejeição da hipótese nula de que o intercepto é zero (Ho: $\beta \mathrm{o}=0)$ indica proximidade do consumo alimentar médio, capturado no QFA e no RA.

Na quinta análise avaliou-se a concordância do consumo de energia, nutrientes e GA através do coeficiente de correlação de concordância (CCC) adaptado por Lin et al. (2012). Nos estudos de validação, o CCC considera a concordância e a correlação de momentos marginais, sendo superior ao coeficiente de correlação de Pearson ( $r$ ) (Lin et al., 2012; Bland \& Altman, 1986). O CCC avalia a acurácia entre dois métodos por uma medida de afastamento da reta estimada por um modelo de regressão linear, entre o consumo alimentar pelos métodos QFA e RA. Se houver concordância completa, espera-se que a reta ajustada esteja posicionada a $45^{\circ}$ da origem, reta chamada linha de concordância, e avalia a precisão pela aferição do quanto cada observação desvia a partir da linha ajustada. O CCC varia de 0 a +1 e McBride (McBride, 2005) propôs uma classificação em que $\mathrm{CCC}<0,90$ denota concordância fraca.

Calibração do QFA. Um modelo de regressão linear misto (com intercepto aleatório) foi utilizado para corrigir os dados de ingestão, tendo as variáveis de consumo alimentar dos RA transformadas, e ajustadas pela energia (nutrientes), como variáveis dependentes e as do QFA como independentes. A inclinação da reta da regressão $(\lambda)$ é a informação-chave para correção do erro da relação entre o consumo mensurado no método referência (RA) e no QFA (Slater et al., 2007) e é chamado de fator de atenuação (Kipnis et al., 2003) ou de calibração. Espera-se que $\lambda$ seja igual a 1,0 e o intercepto $(\alpha)$ aproximadamente zero. Além das estimativas pontuais dos parâmetros deste modelo, seus correspondentes intervalos de $95 \%$ de confiança também são apresentados. Os valores de energia, nutrientes e GA calibrados foram obtidos utilizando-se $\alpha$ e $\lambda$ através da equação: QFA calibrado $=\alpha+\lambda \mathrm{QFA}$.

Adotou-se nível de significância de 5\% para todas as análises. Os dados foram analisados com auxílio do STATA (Stata Corporation, EEUU) versão 12.0.

Aspectos éticos. Pais ou responsáveis pelo estudante menor de idade assinaram o Termo de Consentimento Livre e Esclarecido, e os analfabetos o fizeram por meio da impressão digital. O Prose teve seu protocolo aprovado pelo Comitê de Ética e Pesquisa da UFBA (CAAE 09709112.3.0000.5030/Parecer 182.685 de 04.01.13).

\section{Resultados}

A amostra tem predominância do sexo feminino (79\%), com média de idade de 15,6 anos (DP:1,11) e eutrófica (81\%). Antes das análises de validação aferiu-se o quanto da variação total decorreu das diferenças entre os conglomerados (escolas) através da estimação do ICC (Tabelas 1 e 2) e correspondentes efeitos de desenho, substituindo-se os dados dos ICCs na fórmula do deff. Destas análises, verificou-se que o efeito do desenho foi aproximadamente zero para o consumo de macronutrientes, fibras (ajustado pela energia), e para quatro GA (carnes, leite e derivados, in natura e ultraprocessados). Para cálcio, sódio, zinco e AcGS, ajustados pela energia, por sua vez, o efeito de desenho, obtido variou entre 1,3 a 2,1, e para quatro GA (cereais não integrais, leguminosas, arroz e feijão e processados) variou entre 1,06 a 2,38, justificando a necessidade de correção das análises devido à estrutura de dependência dos dados. 
Tabela 1. Coeficiente de correlação intraclasse (ICC) do modelo não condicional para mensuração do efeito do desenho do estudo sobre as variáveis de consumo do registro alimentar: nutrientes. Prose, 2013/2014.

\begin{tabular}{lcc}
\hline Energia e nutrientes & Modelo não condicional $\dagger$ & $\begin{array}{c}\text { Modelo não condicional } \\
\text { (ajuste por energia) } \dagger^{* *}\end{array}$ \\
\hline Energia (kcal) & 0,0063 & - \\
Proteína (g) & 0,0000 & 0,0000 \\
Gordura total (g) & 0,0000 & 0,0000 \\
Carboidrato (g) & 0,0036 & 0,0000 \\
Fibras (g) & 0,0245 & 0,000 \\
Cálcio (mg) & 0,0426 & 0,0439 \\
Sódio (mg) & 0,0606 & 0,1357 \\
Zinco (mg) & 0,0580 & 0,1412 \\
Ac. g. saturado (g) & 0,0000 & 0,0548 \\
\hline
\end{tabular}

Energia em kcal, variável bruta. †Variáveis transformadas usando raiz quadrada. †*Modelo com nutrientes dos registros alimentares, transformados, ajustados pela energia e com normalidade univariada ( $\mathrm{p}$-valor $>0,05)$. Fonte: Autores.

Tabela 2. Coeficiente de correlação intraclasse (ICC) do modelo não condicional para mensuração do efeito do desenho do estudo sobre as variáveis respostas do registro alimentar: grupos alimentares. Prose, 2013/2014.

\begin{tabular}{lc}
\hline Grupos alimentares & Modelo não condicional $\dagger$ \\
\hline Carnes $(\mathrm{g})$ & 0,0000 \\
Cereais não integrais $(\mathrm{g})$ & 0,0079 \\
Leguminosas $(\mathrm{g})$ & 0,0286 \\
Leite e derivados $(\mathrm{mL})$ & 0,0000 \\
Arroz e feijão $(\mathrm{g})$ & 0,0997 \\
In natura $(\mathrm{g})^{\mathrm{i}}$ & 0,0000 \\
Ultraprocessado $(\mathrm{g})^{\mathrm{i}}$ & 0,0000 \\
Processados $(\mathrm{g})^{\mathrm{i}}$ & 0,1729 \\
\hline
\end{tabular}

$†$ Variáveis transformadas usando raiz quadrada. Modelo com grupos de alimentos dos registros alimentares com normalidade univariada ( $\mathrm{p}$-valor $>0,05)$. ${ }^{\mathrm{i}}$ Grupos baseados no estudo de Louzada et al. (2015). Fonte: Autores.

As diferenças das médias do consumo dos GA $(\mathrm{g} / \mathrm{mL} / \mathrm{dia})$ foram maiores para frutas $(386,9)$ e vegetais $(183,7)$, seguido das bebidas açucaradas $(165,9)$ e café $(193,0)$. As menores discrepâncias médias $(\mathrm{g} / \mathrm{mL} / \mathrm{dia})$ foram encontradas nos óleos e gorduras $(0,78)$, embutidos $(2,4)$, fast food e patissaria $(19,6)$ e peixes e mariscos $(21,0)$. Para o grupo das bebidas alcóolicas não houve superestimação do QFA (-4,3 mL/dia). Dentre os grupos propostos por Louzada et al. (2015), os processados apresentaram a menor diferença entre as médias de consumo $(59,9 \mathrm{~g} /$ dia $)$. A classificação do consumo dos GA entre os adolescentes identificou um consumo regular e concordante entre os dois métodos para o grupo dos cereais não integrais (100\%), seguido das carnes e doces e açucarados ( $99 \%$ cada). Os grupos arroz e feijão, e óleos e gorduras também faziam parte do consumo habitual dos adolescentes (97\%). A concordância para o não consumo foi maior para as bebidas alcóolicas (88\%) e peixes e mariscos (51\%). Leite e derivados apresentaram maior percentual de omissão no QFA e preparações gordurosas (39\%) maior percentual de intrusão (Tabela 3). 
Tabela 3 - Análises descritivas dos grupos alimentares (média e DP) e o percentual de concordância, omissão e intrusão entre consumidores e não consumidores, na comparação do QFA e dos registros alimentares, aplicados a adolescentes do Prose, $2013 / 2014$

\begin{tabular}{|c|c|c|c|c|c|c|}
\hline \multirow[b]{2}{*}{ Grupos alimentares* } & \multicolumn{2}{|c|}{ Média (DP) } & \multicolumn{4}{|c|}{ Concordância $(\%) \dagger$} \\
\hline & $\begin{array}{l}\text { Registro } \\
\text { alimentar }\end{array}$ & QFA & $\begin{array}{c}\text { Não } \\
\text { consome }\end{array}$ & Consome & $\begin{array}{l}\text { Omissão } \\
(\%) \dagger\end{array}$ & Intrusão(\%) \\
\hline Bebida alcoólica (mL) & $12,8(61,3)$ & $8,5(50,1)$ & 88 & 0 & 6 & 6 \\
\hline Bebida açucarada (mL) & $222,8(225,8)$ & $388,6(998,5)$ & 4 & 64 & 12 & 20 \\
\hline Carnes e ovos (g) & $141,3(95,5)$ & $209,8(178,4)$ & 0 & 99 & 0 & 1 \\
\hline $\begin{array}{l}\text { Cereais não integrais } \\
(\mathrm{g})^{* *}\end{array}$ & $307,4(140,4)$ & $338,9(229,0)$ & 0 & 100 & 0 & 0 \\
\hline Doces e açucarados $(\mathrm{g})$ & $75,7(75,5)$ & $161,8(127,9)$ & 0 & 99 & 1 & 0 \\
\hline Embutidos (g) & $8,0(13,8)$ & $10,4(23,4)$ & 28 & 26 & 13 & 33 \\
\hline Fast food/patissaria (g) & $53,9(55,3)$ & $73,5(104,3)$ & 3 & 68 & 14 & 14 \\
\hline Frutas (g) & $148,3(202,2)$ & $535,2(502,9)$ & 1 & 83 & 1 & 14 \\
\hline Vegetais (g) & $52,1(56,5)$ & $235,8(221,1)$ & 1 & 84 & 1 & 13 \\
\hline Óleos e gorduras (g) & $20,7(12,7)$ & $19,9(22,1)$ & 0 & 97 & 3 & 0 \\
\hline Leguminosas $(\mathrm{g})^{* * *}$ & $176,7(140,4)$ & $248,4(254,5)$ & 0 & 91 & 6 & 3 \\
\hline Leite e derivados (mL) & $106,1(101,7)$ & $160,7(194,4)$ & 0 & 91 & 9 & 0 \\
\hline Açúcar simples (g) & $30,6(33,1)$ & $58,5(68,8)$ & 0 & 94 & 6 & 0 \\
\hline Diversos $(\mathrm{g})^{!}$ & $35,8(98,9)$ & $87,4(110,4)$ & 13 & 30 & 3 & 54 \\
\hline Café $(\mathrm{mL}) ! !$ & $151,5(131,5)$ & $344,6(803,9)$ & 12 & 77 & 10 & 1 \\
\hline Sucos de frutas (mL) & $156,7(177,9)$ & $501,3(539,7)$ & 3 & 64 & 7 & 26 \\
\hline $\begin{array}{l}\text { Preparações gordurosas } \\
(\mathrm{g})^{! ! !}\end{array}$ & $20,4(49,2)$ & $44,8(86,3)$ & 38 & 17 & 6 & 39 \\
\hline Peixes e mariscos $(\mathrm{g})$ & $5,7(17,7)$ & $26,8(53,7)$ & 51 & 13 & 1 & 35 \\
\hline Arroz/feijão (g) $)^{! ! ! !}$ & $287,5(212,6)$ & $361,2(290,2)$ & 0 & 97 & 1 & 1 \\
\hline $\begin{array}{l}\text { Leite e deriv. restrito } \\
(\mathrm{mL})^{* !}\end{array}$ & $99,8(97,9)$ & $156,4(192,9)$ & 0 & 86 & 14 & 0 \\
\hline In natura $(\mathrm{g})^{\mathrm{i}^{*}}$ & $1151,94(452,1)$ & $2514,26(1518,9)$ & 1 & 81 & 6 & 12 \\
\hline Ultraprocessado $(\mathrm{g})^{\mathrm{i}^{*}}$ & $461,28(295,1)$ & $761,9(1046,9)$ & 0 & 100 & 0 & 0 \\
\hline Processado $(\mathrm{g})^{\mathrm{i}^{*}}$ & $69,39(57,2)$ & $129,28(118,7)$ & 0 & 100 & 0 & 0 \\
\hline
\end{tabular}

DP: desvio padrão. *Vinte grupos elaborados considerando as características nutricionais dos alimentos e hábitos de consumo: **cereais não integrais, farináceos e amiláceos; ***leguminosas e grãos, cereais integrais e oleaginosas; 'Os alimentos que não se adequaram às demais classificações foram alocados no grupo diversos; !'bebidas não carbonatadas; !"'preparações gordurosas/regionais; !"!! grupo que representa o hábito alimentar brasileiro; ${ }^{*}$ exceto creme de leite/leite condensado. †Classificação baseada no estudo de Vereecken et al. (2005a). ${ }^{\mathrm{i} *}$ Grupos baseados no estudo de Louzada et al. (2015) Fonte: Autoras.

Quanto aos nutrientes, observou-se uma diferença das médias do consumo de energia total entre os dois métodos de $1.269 \mathrm{kcal}$, com superestimação do QFA (média=3.556 kcal, DP=1.407,5) em relação ao RA (média=2.287 kcal, DP=639,0). Os carboidratos e cálcio apresentaram as maiores diferenças entre os macro e micronutrientes, respectivamente. Os limites de concordância (LDC95\%) não se mostraram aceitáveis para os nutrientes, mesmo após o ajuste pela energia. Nenhum dos macronutrientes alcançou concordância exata (100\%), com menor estimativa do QFA para a gordura total e saturada, que ainda 
assim, superestimaram a estimativa dos RA em 1,27 vezes. As maiores diferenças médias entre os dois métodos foram encontradas para fibras (172\%), cálcio (168\%) e carboidratos $(160 \%)$. Os LDC95\% das diferenças observadas entre os métodos não atingiram a faixa de adequação proposta (Ambrosini et al., 2003) e os valores de kappa apresentaram concordância fraca (Landis \& Koch, 1977) $(\mathrm{k}<0,20)$ para a maioria dos nutrientes, à exceção de fibras que apresentou concordância leve (k: $>0,20$ a 0,39$)$ (Tabela 4).

Tabela 4 - Diferenças médias da ingestão de energia e nutrientes e seus respectivos Limites de Concordância 95\% (LDC95\%), e análise de concordância através de tercis das distribuições (Kappa), dos métodos QFA e registros alimentares aplicados a adolescentes escolares do Prose, 2013/2014.

\begin{tabular}{|c|c|c|c|c|c|c|c|c|}
\hline \multirow[b]{2}{*}{ Variáveis } & \multicolumn{2}{|c|}{ Média (DP) } & \multicolumn{3}{|c|}{ Limites de concordância $\dagger$} & \multicolumn{3}{|c|}{ Análise de concordância (Kappa) - Tercis ${ }^{\mathrm{a}}$} \\
\hline & $\begin{array}{l}\text { Registro } \\
\text { alimentar }\end{array}$ & QFA & $\begin{array}{c}\text { Média \% } \\
\text { concordância } \\
(95 \% \mathrm{IC}) ! !\end{array}$ & $\begin{array}{l}\text { LDC } \\
95 \% *\end{array}$ & $\begin{array}{c}\text { LDC } \\
95 \% * *\end{array}$ & $\begin{array}{c}\text { Concordância } \\
\text { bruta }^{\mathbf{b}} \\
(\%)\end{array}$ & $\begin{array}{l}\text { Opostos }^{\mathrm{c}} \\
(\%)\end{array}$ & $\begin{array}{c}k \\
(95 \% \mathrm{IC})\end{array}$ \\
\hline $\begin{array}{l}\text { Energia } \\
\text { (kcal) }\end{array}$ & $\begin{array}{l}2287,5 \\
(639,0)\end{array}$ & $\begin{array}{c}3556,5 \\
(1407,5)\end{array}$ & $\begin{array}{c}148,1 \\
(136,5-160,7)\end{array}$ & $62-355$ & - & - & - & - \\
\hline Proteína (g) & $85,4(31,1)$ & $134,7(64,6)$ & $\begin{array}{c}149,2 \\
(132,9-167,4)\end{array}$ & $48-462$ & $74-302$ & 41 & 28 & $0,12(-0,06-0,29)$ \\
\hline $\begin{array}{l}\text { Gordura } \\
\text { total (g) }\end{array}$ & $74,4(24,9)$ & $101,5(48,9)$ & $\begin{array}{c}127,3 \\
(111,7-145,1)\end{array}$ & $41-391$ & $71-229$ & 38 & 32 & $0,06(-0,1-0,23)$ \\
\hline $\begin{array}{l}\text { Carboidrato } \\
\text { (g) }\end{array}$ & $\begin{array}{c}317,9 \\
(100,3)\end{array}$ & $\begin{array}{c}539,1 \\
(245,3)\end{array}$ & $\begin{array}{c}160,5 \\
(149,3-172,6)\end{array}$ & $61-420$ & + & 39 & 34 & $0,08(-0,03-0,19)$ \\
\hline Fibras (g) & $27,3(14,0)$ & $48,5(27,5)$ & $\begin{array}{c}171,5 \\
(140,8-208,9)\end{array}$ & $47-629$ & $60-495$ & 48 & 24 & $0,22(-0,02-0,47)$ \\
\hline Cálcio (mg) & $\begin{array}{c}399,7 \\
(182,8)\end{array}$ & $\begin{array}{c}723,5 \\
(416,4)\end{array}$ & $\begin{array}{c}168,3 \\
(135,7-208,7)\end{array}$ & $45-624$ & $58-485$ & 43 & 24 & $0,15(0,02-0,29)$ \\
\hline Sódio (mg) & $\begin{array}{c}5058,5 \\
(2158,7)\end{array}$ & $\begin{array}{c}3169,6 \\
(1740,6)\end{array}$ & + & + & + & 38 & 33 & $0,06(-0,08-0,21)$ \\
\hline Zinco (mg) & $10,8(5,3)$ & $16,2(8,6)$ & $\begin{array}{c}144,8 \\
(125,7-166,8)\end{array}$ & $40-527$ & $61-343$ & 43 & 25 & $0,1(-0,08-0,39)$ \\
\hline $\begin{array}{l}\text { Ac. g. } \\
\text { saturado (g) }\end{array}$ & $24,5(9,1)$ & $33,9(17,2)$ & $\begin{array}{c}127,6 \\
(109,7-148,4)\end{array}$ & $37-438$ & $61-268$ & 43 & 32 & $0,15(0,02-0,28)$ \\
\hline
\end{tabular}

DP: desvio padrão das diferenças. †Análise de Bland \& Altman (1986) somente para energia e nutrientes com dados log transformados. !! representa d ou a média de concordância percentual após aplicação do anti $\log (\operatorname{Exp}(d) \times 100)$, onde d = viés ou as diferenças sistemáticas entre os métodos (log QFA - log registro alimentar). 95\%IC: intervalo de confiança para as diferenças médias. ${ }^{*}$ LDC95\%: limites de concordância 95\% $=\mathrm{d}+1.96 * \mathrm{DP}$ com aplicação da função inversa do logaritmo (anti log) aos limites superiores e inferiores (LDC95\%) e expressos em \%; Limites aceitáveis entre $50 \%$ e $200 \% 36$. * LDC95\% dos nutrientes ajustados pela energia. + diferença média não aderiu à distribuição normal e não foi incluído na análise do LDC95\%. ${ }^{\text {a } V a l o r e s ~ d e ~ K a p p a ~}(k)$ para os nutrientes ajustados pela energia. ${ }^{\text {bPercentual }}$ de concordância exata; 'Percentual de classificação no tercil oposto. Todas as análises foram realizadas considerando a dependência dos dados de estudos com desenho amostral complexo. Fonte: Autores.

Os resultados do teste de hipóteses para comparação das diferenças médias de consumo entre os métodos foram estatisticamente significantes para energia e nutrientes avaliados ( $\mathrm{p}$-valor $<0,05$ ). Entre os GA, observou-se que quatro dentre os doze testados foram similares entre os métodos: fast food, leguminosas, leite e derivados, e o grupo arroz e feijão (pvalor $>0,05$ ) (Tabela 5). 
Tabela 5. Avaliação da concordância entre as diferenças das médias do consumo de energia, nutrientes e grupos alimentares (Ho: $\beta_{\mathrm{o}}=0$ ) obtidas através do QFA e dos registros alimentares aplicados a adolescentes escolares do Prose, $2013 / 2014$.

\begin{tabular}{|c|c|c|c|}
\hline Variáveis† $†$ & & $\begin{array}{c}\text { Diferença média } \\
\text { (DP)! }\end{array}$ & $\begin{array}{l}\text { 95\%IC da } \\
\text { regressão* }\end{array}$ \\
\hline Energia (kcal) & $t$ & $11,04(11,4)$ & $9,00-13,08$ \\
\hline \multirow{2}{*}{ Proteína (g) } & $t$, & $2,18(2,9)$ & $1,58-2,79$ \\
\hline & $t j$ & $2,18(1,9)$ & $1,77-2,59$ \\
\hline \multirow{2}{*}{ Gordura total (g) } & $t$, & $1,27(2,5)$ & $0,72-1,83$ \\
\hline & $t j$ & $1,27(1,4)$ & $0,94-1,61$ \\
\hline \multirow{2}{*}{ Carboidrato (g) } & $t$, & $5,02(4,9)$ & $4,22-5,82$ \\
\hline & $t j$ & $5,02(2,3)$ & + \\
\hline \multirow{2}{*}{ Fibras $(\mathrm{g})$} & $t$, & $1,64(1,8)$ & $1,14-2,13$ \\
\hline & $t j$ & $1,64(1,5)$ & $1,23-2,04$ \\
\hline \multirow{2}{*}{ Cálcio (mg) } & $t$, & $6,31(7,6)$ & $3,88-8,73$ \\
\hline & $t j$ & $6,31(6,3)$ & $4,36-8,25$ \\
\hline \multirow{2}{*}{ Sódio (mg) } & $t$, & $-15,54(19,9)$ & $-21,81--9,27$ \\
\hline & $t j$ & $-15,54(16,7)$ & $-21,03--10,05$ \\
\hline \multirow{2}{*}{ Zinco (mg) } & $t$, & $0,68(1,1)$ & $0,43-0,94$ \\
\hline & $t j$ & $0,68(0,8)$ & $0,52-0,85$ \\
\hline \multirow{2}{*}{ Ac. g. saturado (g) } & $t$, & $0,75(1,5)$ & $0,39-1,12$ \\
\hline & $t j$ & $0,75(1,0)$ & $0,46-1,04$ \\
\hline Carnes e ovos (g) & $t$ & $2,22(6,5)$ & $0,59-3,86$ \\
\hline Doces e açucarados (g) & $t$ & $4,03(5,3)$ & $2,99-5,07$ \\
\hline Fast food/patissaria (g) & $t$ & $0,73(5,5)$ & $-1,09-2,55$ \\
\hline Frutas $(\mathrm{g})$ & $t$ & $10,93(10,5)$ & $8,88-12,97$ \\
\hline Leguminosas (g) & $t$ & $1,58(8,2)$ & $-0,82-3,98$ \\
\hline Leite e derivados $(\mathrm{g})$ & $t$ & $1,32(8,2)$ & $-1,4-4,05$ \\
\hline Açúcares (g) & $t$ & $1,51(4,7)$ & $0,78-2,24$ \\
\hline Diversos (g) & $t$ & $4,64(5,9)$ & $3,34-5,95$ \\
\hline Sucos de frutas $(\mathrm{g})$ & $t$ & $9,31(12,8)$ & $6,7-11,91$ \\
\hline Arroz/feijão (g) & $t$ & $1,50(8,1)$ & $-0,63-3,63$ \\
\hline In natura $(\mathrm{g})^{\mathrm{i}}$ & $t$ & $15,0(12,0)$ & $12,25-17,76$ \\
\hline Processado $(\mathrm{g})^{\mathrm{i}}$ & $t$ & $2,69(5,6)$ & $1,83-3,55$ \\
\hline
\end{tabular}

†Variáveis transformadas usando raiz quadrada que aderiram à distribuição normal. $t$ : variáveis transformadas. $t j$ : variáveis transformadas e ajustadas pela energia. ! Diferenças das médias da ingestão de energia, nutrientes e grupos alimentares, entre o QFA e os registros alimentares. 95\% IC: Intervalo de confiança para o intercepto na regressão das diferenças. *Teste de hipótese de diferenças das médias entre os métodos, via modelo linear misto sem covariáveis $(\mathrm{H} 0: \mathrm{QFA}-\mathrm{RA}=0)$. + diferença não aderiu à distribuição normal. íGrupos baseados no estudo de Louzada et al. (2015). Todas as análises foram realizadas considerando a dependência dos dados de estudos com desenho amostral complexo. Fonte: Autores.

O CCC de energia, nutrientes e GA ficou abaixo de 0,9 (McBride, 2005). Dentre os nutrientes, maiores coeficientes foram encontrados para fibras $(\mathrm{CCC}=0,27 ; 95 \% \mathrm{IC}: 0,18-0,35)$, zinco $(\mathrm{CCC}=0,20 ; 95 \% \mathrm{IC}: 0,06-0,34)$ e $\mathrm{AcGS}(\mathrm{CCC}=0,21$; 
95\%IC:0,051-0,36). O ajuste pela energia diminuiu os coeficientes dos macronutrientes, sódio e cálcio (p-valor >0,05). Fibras, zinco e AcGS, ajustados pela energia, mantiveram a significância estatística ( $p$-valor $<0,05$ ) e seus coeficientes variaram de 0,16 a 0,22. Para os sete GA incluídos nessa análise, o coeficiente variou de 0,07 para vegetais $(95 \% \mathrm{IC}:-0,02-0,15)$ a 0,36 para cereais não integrais (95\%IC:0,16-0,56). Os grupos baseados em Louzada e colaboradores (2015) acompanharam os demais (In natura: $\mathrm{CCC}=0,19,95 \% \mathrm{IC}: 0,17-0,21$; Processado: $\mathrm{CCC}=0,27,95 \% \mathrm{IC}: 0,08-0,46$ ) (Tabela 6).

Tabela 6. Coeficiente de correlação de concordância de energia, nutrientes e grupos alimentares do QFA e do método referência considerando o efeito do desenho do estudo. Prose, 2013/2014.

\begin{tabular}{|c|c|c|c|}
\hline Variáveis $\dagger$ & & CCC (EP) & $95 \% \mathrm{IC}$ \\
\hline \multirow{2}{*}{ Energia (kcal) } & $\mathrm{CCC}_{\text {bruta }}$ & $0,18(0,04)$ & $0,11-0,25$ \\
\hline & $\mathrm{CCC}_{\mathrm{t}}$ & $0,20(0,04)$ & $0,11-0,28$ \\
\hline \multirow{2}{*}{ Proteína (g) } & $\mathrm{CCCt}$ & $0,14(0,04)$ & $0,07-0,22$ \\
\hline & $\mathrm{CCCtj}$ & $0,07(0,06)$ & $-0,05-0,19$ \\
\hline \multirow{2}{*}{ Gordura total (g) } & $\mathrm{CCCt}$ & $0,19(0,07)$ & $0,053-0,32$ \\
\hline & $\mathrm{CCCtj}$ & $0,08(0,07)$ & $-0,05-0,21$ \\
\hline \multirow{2}{*}{ Carboidrato (g) } & $\mathrm{CCCt}$ & $0,18(0,07)$ & $0,05-0,31$ \\
\hline & $\mathrm{CCCtj}$ & $0,01(0,02)$ & $-0,03-0,05$ \\
\hline \multirow{2}{*}{ Fibras (g) } & $\mathrm{CCCt}$ & $0,27(0,04)$ & $0,19-0,36$ \\
\hline & $\mathrm{CCCtj}$ & $0,17(0,07)$ & $0,03-0,31$ \\
\hline \multirow{2}{*}{ Cálcio (mg) } & $\mathrm{CCCt}$ & $0,18(0,04)$ & $0,10-0,27$ \\
\hline & $\mathrm{CCCtj}$ & $0,11(0,06)$ & $-0,01-0,22$ \\
\hline \multirow{2}{*}{ Sódio (mg) } & $\mathrm{CCCt}$ & $0,10(0,06)$ & $-0,02-0,22$ \\
\hline & $\mathrm{CCCtj}$ & $-0,02(0,06)$ & $-0,13-0,10$ \\
\hline \multirow{2}{*}{ Zinco (mg) } & $\mathrm{CCCt}$ & $0,20(0,07)$ & $0,06-0,34$ \\
\hline & CCCtj & $0,22(0,07)$ & $0,08-0,36$ \\
\hline \multirow{2}{*}{ Ácidos graxos saturados (mg) } & $\mathrm{CCCt}$ & $0,21(0,08)$ & $0,05-0,36$ \\
\hline & $\mathrm{CCCtj}$ & $0,16(0,07)$ & $0,04-0,29$ \\
\hline Cereais não integrais (g) & $\mathrm{CCCt}$ & $0,36(0,10)$ & $0,16-0,56$ \\
\hline Frutas $(\mathrm{g})$ & $\mathrm{CCCt}$ & $0,19(0,09)$ & $0,01-0,36$ \\
\hline Vegetais (g) & $\mathrm{CCCt}$ & $0,07(0,04)$ & $-0,02-0,15$ \\
\hline Leguminosas(g) & $\mathrm{CCCt}$ & $0,29(0,09)$ & $0,11-0,47$ \\
\hline Sucos de frutas $(\mathrm{g})$ & $\mathrm{CCCt}$ & $0,13(0,07)$ & $-0,01-0,27$ \\
\hline Arroz e feijão(g) & $\mathrm{CCCt}$ & $0,34(0,07)$ & $0,20-0,48$ \\
\hline Leite e derivados $(\mathrm{mL})$ & $\mathrm{CCCt}$ & $0,26(0,11)$ & $0,04-0,48$ \\
\hline In natura $(\mathrm{g})^{\mathrm{i}}$ & $\mathrm{CCCt}$ & $0,19(0,01)$ & $0,17-0,21$ \\
\hline Processado $(\mathrm{g})^{\mathrm{i}}$ & $\mathrm{CCCt}$ & $0,27(0,10)$ & $0,08-0,46$ \\
\hline
\end{tabular}

†Todas as variáveis foram transformadas usando raiz quadrada e somente os pares de variáveis com normalidade bivariada foram incluídos nesta análise. EP: erro padrão linearizado. CCC - Coeficiente de Correlação de Concordância. $\mathrm{CCC}_{\mathrm{t}}$ : variável transformada. $\mathrm{CCC}_{\mathrm{tj}}$ : variável transformada e ajustada pela energia. 95\%IC: Intervalo de confiança para o Coeficiente. CCC calculado com base no estudo de Lin et al. (2012). 'Grupos baseados no estudo de Louzada et al. (2015). Todas as análises foram realizadas considerando a dependência dos dados de estudos com desenho amostral complexo. Fonte: Autores.

Menores coeficientes de calibração indicam maior erro de medida na estimativa do QFA. Neste estudo, os 
macronutrientes, ajustados pela energia, apresentaram os menores valores de $\lambda$ (carboidratos: $\lambda=0,05$; gordura total: $\lambda=0,08$ ), valores similares foram também observados para três GA (bebidas açucaradas: $\lambda=0,08$; carnes: $\lambda=0,07$; vegetais: $\lambda=0,09$ ). Entre os nutrientes, o zinco obteve o maior fator após o ajuste por energia $(\lambda=0,31)$ e, entre os GA, o arroz e feijão, e cereais não integrais tiveram melhor desempenho ( $\lambda=0,28$ e $\lambda=0,26$, respectivamente) (Tabela 7$)$.

Tabela 7. Parâmetros de calibração $\alpha$ e $\lambda$ de energia, dos nutrientes ajustados pela energia e grupos alimentares do QFA, e intervalos de confiança (95\%IC) associados. Prose 2013/2014.

\begin{tabular}{|c|c|c|}
\hline Variáveis & $\alpha(95 \% \mathrm{IC})$ & $\lambda(95 \% \mathrm{IC})$ \\
\hline \multicolumn{3}{|l|}{ Energia e nutrientes } \\
\hline Energia $(\mathrm{kcal}) \dagger$ & $1630,31(1465,03-1795,60)$ & $0,18(0,11-0,25)$ \\
\hline Proteína (g) & $7,79(4,95-10,63)$ & $0,12(-0,14-0,37)$ \\
\hline Gordura total (g) & $7,73(6,17-9,23)$ & $0,08(-0,07-0,23)$ \\
\hline Carboidrato (g) & $16,49(12,87-20,12)$ & $0,05(-0,11-0,21)$ \\
\hline Fibras (g) & $3,42(1,99-4,85)$ & $0,24(0,03-0,46)$ \\
\hline Cálcio (g) & $15,89(10,43-21,35)$ & $0,14(-0,04-0,32)$ \\
\hline Zinco (g) & $1,99(1,13-2,84)$ & $0,31(0,08-0,54)$ \\
\hline Ac. gx. Saturado (g) & $3,93(3,07-4,79)$ & $0,17(0,02-0,31)$ \\
\hline \multicolumn{3}{|l|}{ Grupos alimentares } \\
\hline Bebidas açucaradas (mL) & $10,93(6,25-15,61)$ & $0,08(-0,13-0,3)$ \\
\hline Carnes (g) & $10,19(7,03-13,34)$ & $0,07(-0,13-0,28)$ \\
\hline Cereais não integrais (g) & $12,63(9,57-15,70)$ & $0,26(0,08-0,43)$ \\
\hline Fast food (g) & $4,68(3,23-6,12)$ & $0,22(0,11-0,32)$ \\
\hline Vegetais (g) & $4,68(3,23-6,12)$ & $0,09(-0,03-0,22)$ \\
\hline Leguminosas (g) & $8,97(5,13-12,81)$ & $0,23(0,04-0,41)$ \\
\hline Leite $(g)$ & $6,74(3,70-9,79)$ & $0,20(-0,01-0,41)$ \\
\hline Sucos de frutas $(\mathrm{mL})$ & $7,21(3,52-10,90)$ & $0,13(-0,01-0,28)$ \\
\hline Arroz e feijão (g) & $10,93(7,05-14,81)$ & $0,28(0,12-0,44)$ \\
\hline In natura $(g)^{i}$ & $22,09(18,18-26,00)$ & $0,23(0,16-0,30)$ \\
\hline Ultraprocessado $(\mathrm{g})^{\mathrm{i}}$ & $15,21(13,10-17,33)$ & $0,21(0,13-0,28)$ \\
\hline Processado $(\mathrm{g})^{\mathrm{i}}$ & $4,94(2,93-6,96)$ & $0,24(0,11-0,36)$ \\
\hline
\end{tabular}

$\dagger$ Energia bruta. Energia, nutrientes e grupos alimentares transformados usando raiz quadrada. Nutrientes transformados e ajustados pela energia. $\alpha(95 \% \mathrm{IC})=$ intercepto da regressão e respectivos intervalos de confiança; $\lambda(95 \% \mathrm{IC})=$ inclinação da reta da regressão estimada e respectivos intervalos de confiança. ${ }^{\mathrm{i}}$ Grupos baseados no estudo de Louzada et al. (2015). Todas as análises foram realizadas considerando a dependência dos dados de estudos com desenho amostral complexo. Fonte: Autores.

\section{Discussão}

Diversas estratégias foram utilizadas para avaliar a validade e estimar os fatores de calibração de um QFA aplicado a adolescentes escolares, considerando o efeito do desenho amostral complexo. O efeito da escola (conglomerado) sobre o consumo alimentar foi capturado através da estimação do ICC e correspondentes efeitos de desenho (deff) e os resultados mostraram que a estrutura de dependência dos dados é relevante na análise de metade dos nutrientes e dos GA, além da energia total, mesmo em um estudo de validação onde o tamanho amostral é usualmente menor. A estrutura de dependência relativa 
aos dados de consumo alimentar de estudantes das dez escolas foi incorporada em todas as análises, mesmo para aquelas variáveis onde o ICC foi aproximadamente zero. O ajuste das análises pelo desenho do estudo não altera as estimativas pontuais, mas pode influenciar os procedimentos de comparação dos dois métodos de obtenção do consumo alimentar, sobretudo na realização de testes de hipóteses e definição de intervalos de confiança, não podendo, por esta razão, ser ignorado.

As análises evidenciaram a validade relativa do QFA. Percentualmente, identificou-se concordância de aproximadamente $100 \%$ entre os métodos para o consumo de alimentos fontes de carboidratos refinados (cereais não integrais, doces e açucarados) e carnes, antecipando uma tendência para um padrão alimentar comum entre adolescentes (WHO, 2005; Vieira, Del Ciampo \& Del Ciampo, 2014). A concordância dos métodos foi ratificada na análise da comparação de médias para 33\% dos GA (p-valor >0,05), resultado similar ao encontrado em estudo com adolescentes (Voci, Enes, \& Slater, 2008) que validou um QFA, obtendo boa validade para $28 \%$ dos GA avaliados. Porém, quando o consumo entre os dois métodos foi testado através da ingestão de energia e nutrientes, encontrou-se diferenças significantes ( $\mathrm{p}$-valor $<0,05$ ), resultados compatíveis com estudo realizado no mesmo estado (Matos et al., 2012). Possível explicação para essas diferenças seria um número insuficiente de RA para avaliar os nutrientes, pois erros aleatórios aumentam a variância das estimativas de consumo dietético, com consequente redução da precisão, e o efeito desses erros pode ser reduzido pelo aumento do número de observações (Rutishauser, 2005). Realizou-se análise de sensibilidade comparando-se os resultados obtidos com as médias dos três RA (Tabela 3) e os obtidos pelo método Multiple Source Method (MSM), que é usualmente indicado para corrigir a variabilidade intrapessoal, e não encontramos diferenças a respeito dos resultados apresentados anteriormente, mantendo-se as diferenças significantes entre os nutrientes obtidos nos dois métodos (dados não mostrados). Ressalte-se que nesta investigação foram coletados três RA por indivíduo, quantidade comum em estudos de validação. Ainda que possam não representar a ingestão dietética total, as repetições do RA permitiram capturar o consumo alimentar da semana, conforme planejado pelo estudo.

A medida de concordância utilizada (CCC) aplica-se a estudos com amostragem complexa. Sua robustez frente ao $r$ (Lin et al., 2012) e às críticas sobre a indevida interpretação do $r$ em estudos que avaliam concordância entre dois métodos, como os estudos de validação (Bland \& Altman, 1986) ratificaram seu uso. Considerando a classificação do CCC (McBride, 2005), os métodos mostraram concordância fraca para o consumo dos nutrientes e GA, com melhores resultados para leguminosas $(\mathrm{CCC}=0,29)$, leite e derivados $(\mathrm{CCC}=0,26)$, arroz e feijão $(\mathrm{CCC}=0,34)$ e processados $(\mathrm{CCC}=0,27)$. Entretanto, a interpretação proposta por McBride (2005) é bem restrita quando comparada à comumente utilizada para o coeficiente de correlação de Pearson (<0,2: fraca e >0,8: excelente em algumas situações) (Altman, 1991), estando a concordância encontrada neste intervalo $(0,2<\mathrm{CCC}<0,8)$ para cinco GA. A diminuição do CCC para os nutrientes ajustados pela energia pode ser explicada pela superestimação encontrada no QFA, mas não foram encontrados estudos de validação com o CCC para comparação dos resultados. Entretanto, estudos com adolescentes relataram diminuição dos coeficientes de correlação após o ajustamento (Matos et al., 2012; Henn et al., 2010). Uma das justificativas para realizar o ajuste pela energia total é o controle do confundimento, sobretudo se o consumo de energia total está associado a fatores como atividade física, composição corporal e eficiência metabólica (Willett, Howe \& Kushi, 1997), não sendo aqui avaliado. Os GA não foram ajustados pela energia, pois optou-se por utilizar o perfil de grupos e não sua composição centesimal. Decisão baseada na tendência de uso de frutas (Vereecken et al., 2005b; Moore et al., 2012; Alves et al., 2021), hortaliças (Moore et al., 2012; Alves et al., 2021), alimentos processados ou ultraprocessados (Louzada et al., 2015; Vereecken et al., 2005b) para avaliar as relações com desfechos em saúde.

Ainda assim, o QFA superestimou o consumo de nutrientes, resultado compatível com outros estudos (Gigante et al., 2010; Matos et al., 2012; Brito et al., 2017). O mesmo comportamento foi verificado para alguns GA (Voci, Enes \& Slater, 
2008), sendo necessário corrigir os dados de consumo através dos fatores de calibração $(\lambda)$ que ratificaram as diferenças entre os métodos uma vez que encontrou-se $\lambda<0,4$. Quanto menor o valor de atenuação pior o desempenho do QFA, e maior atenuação de uma estimativa de risco em uma associação dieta-doença, caso o QFA seja utilizado sem a calibração (Freedman et al., 2011). Os fatores de calibração encontrados em outro estudo com adolescentes (Voci et al., 2011) variaram de 0,07 (ferro) a 0,32 (fibras), próximos aos encontrados neste estudo. Maiores fatores de calibração também foram encontrados em outro estudo com adolescentes, mas com análises estratificadas por sexo ( $\lambda: 0,14$ a 0,48) (Araujo, Yokoo \& Pereira, 2010), diferenças que podem ser originadas dessa estratificação, aqui não realizada.

Observou-se neste e em outros estudos com adolescentes que os fatores de calibração são geralmente pequenos quando comparados aos estudos com adultos, para nutrientes (Johansson et al., 2002; Carithers et al., 2009) e GA (Johansson et al., 2002). Acredita-se que as características próprias da adolescência influenciem as respostas ao QFA, levando a menor concordância entre os métodos e aos fatores de calibração encontrados (Voci et al., 2011; Araujo, Yokoo \& Pereira, 2010).

As diferenças encontradas e ratificadas em estudos com adolescentes corroboram as conhecidas limitações do QFA e a necessidade de sua validação para uso na identificação de associações com desfechos em saúde (Cade et al., 2004). Porém, a presença de erros sistemáticos advindos da população do estudo não pode ser descartada, considerando que adolescentes podem ter dificuldades em relembrar seu consumo, necessitando de motivação para o preenchimento dos RA (Thompson \& Subar, 2013) logo após as refeições, além do consumo fora do lar. Para minimizar esses erros, um estudo piloto em Salvador/Bahia testou o QFA com adolescentes escolares de uma escola pública não incluída na amostra, possibilitando a revisão do quantitativo de itens e a abordagem com os escolares; utilizou-se álbum fotográfico com porções dos alimentos; orientações foram fornecidas pela equipe de campo; a aplicação do QFA antecedeu ao método referência (Cade et al., 2004) e o tamanho amostral do estudo atendeu as recomendações da literatura (Cade et al., 2004; Voci et al., 2011) sendo proveniente de uma amostra probabilística em conglomerados.

Entre as limitações deste estudo destacamos a dificuldade em qualificar os itens do QFA na classificação NOVA (Louzada et al., 2015), pois não há, por exemplo, detalhes sobre as preparações mistas. Entende-se, porém, que tais dificuldades não inviabilizam o uso da classificação nos estudos de validação. Outras questões referem-se à utilização dos RA como método referência, que apresenta limitações quando comparado aos com maior acurácia e com menor erro de mensuração, além da não avaliação do método referência para identificar se era livre de erros e dos relacionados ao QFA por meio, por exemplo, de estudo de comparação com métodos mais acurados (Kipnis et al., 2003; Kobe, Kržišnik \& Mis, 2012). Entretanto, existem limites para aplicação dos métodos mais acurados como alto custo (Thompson \& Subar, 2013), o fato de serem pouco amigáveis e não existirem para todos os fatores dietéticos (Thompson \& Subar, 2013; Willett, 1998). Apesar do Apesar do reconhecimento das limitações dos RA, sua aplicação em estudos de validação de QFA tem plausibilidade cientifica, sendo utilizado em diversos estudos (Mascarenhas et al., 2016; Araujo, Yokoo \& Pereira, 2010).

A cooperação necessária para preenchimento dos RA (Thompson \& Subar, 2013) também deve ser considerada e pode ter limitado o número de respondentes e a generalização dos resultados, pois mesmo tendo-se estimulado um maior número de adolescentes, de ambos os sexos, para participarem do estudo, a presença feminina foi maior. Sabe-se que voluntários em um subestudo, como o de validação, podem responder com maior acurácia do que os sorteados a responder o QFA (Cade et al., 2004). Ainda, na adolescência, as pressões culturais e sociais exercem influência, por exemplo, para seguir um padrão alimentar "correto", onde adolescentes com baixo consumo de alimentos supostamente mais saudáveis tendem a superestimar esse consumo, enquanto indivíduos com alto consumo de alimentos pouco saudáveis tendem ao sub relato desses alimentos (Kipnis et al., 1999). Questões como estas poderiam explicar as diferenças no consumo de frutas e vegetais, encontradas entre os dois métodos, reforçando a necessidade de validação e calibração do QFA.

Estudar o consumo alimentar de adolescentes é um desafio, em especial a validação de instrumento de mensuração 
desse consumo, considerando tanto as características próprias dessa população quanto as dificuldades em se mensurar esse complexo construto. A validade relativa do QFA utilizado foi comprovada através de várias análises e o uso do $\lambda$ permite melhorar a acurácia do QFA, incluindo o desenho amostral do estudo original nesses procedimentos.

\section{Considerações Finais}

As análises apontaram que o QFA superestimou as estimativas de consumo quando comparadas ao método referência, principalmente quando avaliada a ingestão de nutrientes. Porém, as diferenças observadas para alguns GA não foram estatisticamente significantes, havendo ainda concordância entre os métodos para o consumo de alimentos fontes de carboidratos refinados, gorduras, carnes e o grupo arroz e feijão.

O QFA apresentou validade relativa, principalmente quando avaliado através dos GA. Para o uso dos nutrientes como preditor em associações com indicadores de saúde, os fatores de calibração devem ser utilizados para aproximar as estimativas do QFA ao método referência.

A investigação de padrões alimentares de adolescentes não é tarefa simples, envolvendo diversas etapas para a adequada validação dos métodos que serão utilizados para gerar esse construto, que não sendo diretamente observado, deve ser bem quantificado. Neste estudo, além da observância das recomendações disponíveis na literatura quanto aos procedimentos de coleta dos dados e aos métodos de análise utilizados, incluiu-se em todas as análises a correção do efeito do desenho do estudo considerando a amostragem em conglomerados. Estes procedimentos não são rotineiramente utilizados em estudos de validação de QFA com amostras complexas que, frequentemente, analisam os dados como se fossem provenientes de amostragem aleatória simples.

Dada a necessidade de validação dos instrumentos de avaliação do consumo alimentar para populações específicas e a crescente utilização de pesquisas com desenho amostral complexo, sugere-se para investigações futuras, a incorporação de procedimentos adequados para validar métodos de mensuração, incluindo os ajustes necessários ao plano amostral.

\section{Agradecimentos}

Aos componentes do Programa Integrado de Pesquisa e Cooperação Técnica em Formação e Avaliação da Atenção Básica (GRAB), aos dirigentes escolares que apoiaram o Prose e aos adolescentes escolares que participaram da pesquisa.

\section{Referências}

Altman, D. G. (1991). Practical statistics for medical research. CRC press.

Alves, S. P. P.; Simões, M. O. S.; Barros, K. N. N. O.; Silva, R. M. P.; Barros, A. M. G.; Medeiros, C. C. M., \& Carvalho, D. F. (2021). Fatores associados à anemia em adolescentes escolares: Um estudo transversal. Research, Society and Development, 10(5), e3310514521.

Ambrosini, G. L., Van Roosbroeck, S. A., Mackerras, D., Fritschi, L., De Klerk, N. H., \& Musk, A. W. (2003). The reliability of ten-year dietary recall: implications for cancer research. The Journal of nutrition, 133(8), 2663-2668.

Araujo, M. C., Veiga, G. V. D., Sichieri, R., \& Pereira, R. A. (2010). Elaboração de questionário de frequência alimentar semiquantitativo para adolescentes da região metropolitana do Rio de Janeiro, Brasil. Revista de Nutrição, 23(2), 179-189.

Araujo, M. C., Yokoo, E. M., \& Pereira, R. A. (2010). Validation and calibration of a semiquantitative food frequency questionnaire designed for adolescents. Journal of the American Dietetic Association, 110(8), 1170-1177.

Baranowski, T. (2012). 24-hour recall and diet record methods. In W. C. Willett (Ed.), Nutritional epidemiology, 4, (p. 49-69).

Beaton, G. H., Burema, J., \& Ritenbaugh, C. (1997). Errors in the interpretation of dietary assessments. The American journal of clinical nutrition, 65(4), $1100 \mathrm{~S}-1107 \mathrm{~S}$.

Bechthold, A., Boeing, H., Schwedhelm, C., Hoffmann, G., Knüppel, S., Iqbal, K., \& Schwingshackl, L. (2019). Food groups and risk of coronary heart disease, stroke and heart failure: a systematic review and dose-response meta-analysis of prospective studies. Critical reviews in food science and nutrition, 59(7), 1071-1090. 
Bell, B. A., Onwuegbuzie, A. J., Ferron, J. M., Jiao, Q. G., Hibbard, S. T., \& Kromrey, J. D. (2012). Use of design effects and sample weights in complex health survey data: a review of published articles using data from 3 commonly used adolescent health surveys. American journal of public health, 102(7), 1399-1405.

Bland, J. M. (2004). Cluster randomised trials in the medical literature: two bibliometric surveys. BMC Medical Research Methodology, 4 (21), 1-6.

Bland, J. M., \& Altman, D. (1986). Statistical methods for assessing agreement between two methods of clinical measurement. The Lancet, 327(8476), 307310 .

Brito, A. P., Araujo, M. C., Guimarães, C. P., \& Pereira, R. A. (2017). Validade relativa de questionário de frequência alimentar com suporte de imagens. Ciência \& Saúde Coletiva, 22, 457-468.

Cade, J. E., Burley, V. J., Warm, D. L., Thompson, R. L., \& Margetts, B. M. (2004). Food-frequency questionnaires: a review of their design, validation and utilisation. Nutrition research reviews, 17(1), 5-22.

Carithers, T. C., Talegawkar, S. A., Rowser, M. L., Henry, O. R., Dubbert, P. M., Bogle, M. L., \& Tucker, K. L. (2009). Validity and calibration of food frequency questionnaires used with African-American adults in the Jackson Heart Study. Journal of the American Dietetic Association, 109(7), $1184-1193$.

Fornés, N. S. D., Martins, I. S., Velásquez-Meléndez, G., \& Latorre, M. D. R. D. D. O. (2002). Escores de consumo alimentar e níveis lipêmicos em população de São Paulo, Brasil. Revista de Saúde Pública, 36(1), 12-18.

Freedman, L. S., Schatzkin, A., Midthune, D., \& Kipnis, V. (2011). Dealing with dietary measurement error in nutritional cohort studies. Journal of the National Cancer Institute, 103(14), 1086-1092.

Furlan-Viebig, R., \& Pastor-Valero, M. (2004). Desenvolvimento de um questionário de freqüência alimentar para o estudo de dieta e doenças não transmissíveis. Revista de Saúde Pública, 38(4), 581-584.

Gigante, D. P., Reichert, F. F., Hallal, P. C., Souza, R. V. D., Neutzling, M. B., Vieira, M. D. F. A., \& Menezes, A. (2010). Dietary assessment in the 1993 Pelotas (Brazil) birth cohort study: comparing energy intake with energy expenditure. Cadernos de saude publica, 26(11), 2080-2089.

Hedges, L. V. (2007). Correcting a significance test for clustering. Journal Educ. Behav. Stat. 32(2), 151-179.

Hedges, L. V., \& Hedberg, E. C. (2007). Intraclass correlation values for planning group-randomized trials in education. Educational Evaluation and Policy Analysis, 29(1), 60-87.

Henn, R. L., Fuchs, S. C., Moreira, L. B., \& Fuchs, F. D. (2010). Development and validation of a food frequency questionnaire (FFQ-Porto Alegre) for adolescent, adult and elderly populations from Southern Brazil. Cadernos de saude publica, 26, 2068-2079.

Instituto Brasileiro de Geografia e Estatística. (2011). Pesquisa de Orçamentos Familiares 2008-2009: tabelas de composição nutricional dos alimentos consumidos no Brasil.

Johansson, I., Hallmans, G., Wikman, Å., Biessy, C., Riboli, E., \& Kaaks, R. (2002). Validation and calibration of food-frequency questionnaire measurements in the Northern Sweden Health and Disease cohort. Public health nutrition, 5(3), 487-496.

Kang, C., Qaqish, B., Monaco, J., Sheridan, S. L., \& Cai, J. (2013). Kappa statistic for clustered dichotomous responses from physicians and patients. Statistics in medicine, 32(21), 3700-3719.

Kipnis, V., Carroll, R. J., Freedman, L. S., \& Li, L. (1999). Implications of a new dietary measurement error model for estimation of relative risk: application to four calibration studies. American Journal of Epidemiology, 150(6), 642-651.

Kipnis, V., Subar, A. F., Midthune, D., Freedman, L. S., Ballard-Barbash, R., Troiano, R. P., \& Carroll, R. J. (2003). Structure of dietary measurement error: results of the OPEN biomarker study. American journal of epidemiology, 158(1), 14-21.

Kobe, H., Kržišnik, C., \& Mis, N. F. (2012). Under-and over-reporting of energy intake in Slovenian adolescents. Journal of nutrition education and behavior, 44(6), 574-583.

Köche, J. C. (2011). Fundamentos de metodologia científica: teoria da ciência e iniciação à pesquisa. Vozes. http://www.adm.ufrpe.br/sites/ww4.deinfo.ufrpe.br/files/Fundamentos_de_Metodologia_Cienti\%CC\%81 fica.pdf

Kouvari, M., Mamalaki, E., Bathrellou, E., Poulimeneas, D., Yannakoulia, M., \& Panagiotakos, D. B. (2020). The validity of technology-based dietary assessment methods in childhood and adolescence: a systematic review. Critical reviews in food science and nutrition, 1-16.

Landis, J. R., \& Koch, G. G. (1977). A one-way components of variance model for categorical data. Biometrics, 33, 671-679.

Lin, H. M., Kim, H. Y., Williamson, J. M., \& Lesser, V. M. (2012). Estimating agreement coefficients from sample survey data. Survey Methodology, 38(1), 63-72.

Lohman, T. J., Roache, A. F., \& Martorell R. (1992). Anthropometric standardization reference manual. Human Kinetics: Champaign IL USA.

Louzada, M. L. D. C., Martins, A. P. B., Canella, D. S., Baraldi, L. G., Levy, R. B., Claro, R. M., \& Monteiro, C. A. (2015). Alimentos ultraprocessados e perfil nutricional da dieta no Brasil. Revista de Saúde Pública, 49(38), 1-11.

Mascarenhas, J. M. O., Silva, R. D. C. R., Machado, M. E. P. C., Santos, C. A. D. S. T., Marchioni, D. M. L., \& Barreto, M. L. (2016). Validation of a food frequency questionnaire designed for adolescents in Salvador, Bahia, Brazil. Revista de Nutrição, 29(2), 163-171.

Matos, S. M. A. D., Prado, M. D. S., Santos, C. A. D. S. T., D'Innocenzo, S., Assis, A. M. D. O., Dourado, L. S., \& Barreto, M. L. (2012). Validation of a food frequency questionnaire for children and adolescents aged 4 to 11 years living in Salvador, Bahia. Nutricion hospitalaria, 27(4), 1114-1119. 
Research, Society and Development, v. 10, n. 6, e5391016075, 2021

(CC BY 4.0) | ISSN 2525-3409 | DOI: http://dx.doi.org/10.33448/rsd-v10i6.16075

McBride, G. B. (2005). A proposal for strength-of-agreement criteria for Lin's Concordance Correlation Coefficient. NIWA, Hamilton. NIWA Client Report: HAM2005-062. Disponível em: https://www.medcalc.org/download/pdf/McBride2005.pdf

Moore, L. L., Bradlee, M. L., Singer, M. R., Qureshi, M. M., Buendia, J. R., \& Daniels, S. R. (2012). Dietary Approaches to Stop Hypertension (DASH) eating pattern and risk of elevated blood pressure in adolescent girls. British journal of nutrition, 108(9), 1678-1685.

Onis, M. D., Onyango, A. W., Borghi, E., Siyam, A., Nishida, C., \& Siekmann, J. (2007). Development of a WHO growth reference for school-aged children and adolescents. Bulletin of the World health Organization, 85, 660-667.

Pinheiro, A. B. V., Lacerda, E. M. D. A., Benzecry, E. H., Gomes, M. C. D. S., \& Costa, V. M. D. (2005). Tabela para avaliação de consumo alimentar em medidas caseiras (5a ed.) São Paulo: Atheneu.

Richmond, T. K., \& Subramanian, S. V. (2008). School level contextual factors are associated with the weight status of adolescent males and females. Obesity, $16(6), 1324-1330$.

Rutishauser, I. H. (2005). Dietary intake measurements. Public health nutrition, 8(7a), 1100-1107.

Slater, B., Marchioni, D. M. L., \& Voci, S. M. (2007). Use of linear regression for correction of dietary data. Revista de saude publica, 41 , 190-196.

Taco - Tabela brasileira de composição de alimentos. (2011). (4a. ed.). NEPA-UNICAMP.

Thompson, F. E., \& Subar, A. F. (2013). Dietary Assessment Methodology. In A. M. Coulston, C. J. Boushey, \& M. G. Ferruzzi (Eds.), Nutrition in the Prevention and Treatment of Disease 2, (3a. ed.). San Diego CA: Academic Press.

Vereecken, C. A., Covents, M., Matthys, C., \& Maes, L. (2005a). Young adolescents' nutrition assessment on computer (YANA-C). European journal of clinical nutrition, 59(5), 658-667.

Vereecken, C. A., Inchley, J., Subramanian, S. V., Hublet, A., \& Maes, L. (2005b). The relative influence of individual and contextual socio-economic status on consumption of fruit and soft drinks among adolescents in Europe. The European Journal of Public Health, 15(3), $224-232$.

Vieira, M. V., Del Ciampo, I. R. L., \& Del Ciampo, L. A. (2014). Hábitos e consumo alimentar entre adolescentes eutróficos e com excesso de peso. Revista Brasileira de Crescimento e Desenvolvimento Humano, 24(2), 157-162.

Voci, S. M., Enes, C. C., \& Slater, B. (2008). Validação do Questionário de Frequência Alimentar para Adolescentes (QFAA) por grupos de alimentos em uma população de escolares. Revista Brasileira de Epidemiologia, 11(4), 561-572.

Voci, S. M., Slater, B., Silva, M. V. D., Marchioni, D. M. L., \& Latorre, M. D. R. D. D. O. (2011). Estudo de calibração do Questionário de Frequência Alimentar para Adolescentes (QFAA). Ciência \& Saúde Coletiva, 16, 2335-2343.

Willett, W. C. (1998). Nutritional epidemiology. Oxford University Press.

Willett, W. C., Howe, G. R., \& Kushi, L. H. (1997). Adjustment for total energy intake in epidemiologic studies. The American journal of clinical nutrition, $65(4), 1220 \mathrm{~S}-1228 \mathrm{~S}$.

Who - World Health Organization. (2005). Nutrition in adolescence: issues and challenges for the health sector: issues in adolescent health and development. https://apps.who.int/iris/bitstream/handle/10665/43342/9241593660_eng.pdf 